\title{
STABILITY ANALYSIS OF POWER CIRCUIT COMPRISING VIRTUAL INDUCTANCE
}

\author{
O. Dranga ${ }^{*}$, H. Funato ${ }^{*}$, S. Ogasawara ${ }^{*}$, C.K. Tse ${ }^{* *}$ and H.H.C. Iu ${ }^{* * *}$ \\ * Department of Electrical and Electronic Engineering, Utsunomiya University, Japan \\ ** Department of Electronic and Information Engineering, Hong Kong Polytechnic University, Hong Kong \\ ${ }^{* * *}$ School of Electrical, Electronic and Computer Engineering, The University of Western Australia, Australia
}

\begin{abstract}
The concept of a variable active-passive reactance (VAPAR), which can generate a virtual variable inductance in power circuits, including negative values, has already been proposed. Since it has found applications in the power flow control of power systems, where the power flow is essentially restricted by a line inductance, the aim of the paper is to report on the stability of this virtual inductance in an RL circuit. The periodic steady-state operation of the variablestructure, piecewise-linear, nonlinear system is modeled by its Poincaré map. The stability criterion employs the position of the eigenvalues of the Jacobian matrix of the Poincaré map, evaluated at its fixed point. Bifurcation behaviour when varying the virtual inductance is revealed. Such an analysis allows accurate prediction of stability boundaries and facilitates the selection of parameter values to guarantee stable operation.
\end{abstract}

\section{INTRODUCTION}

Inductance plays a significant role in power systems: the current response of DC motors depends on the armature inductance, the power flow of power systems depends on the line inductance, the operation of induction motor depends on the rotor leakage inductance, etc. It may therefore represent an important tool for shaping the response of power circuits. A variable active-passive reactance (VAPAR) was proposed in [1] and discussed in a number of publications later [2]-[4] in order to generate a virtual variable inductance, including negative values. It has found applications in the rapid power flow control of power systems, for flexible $\mathrm{AC}$ transmission systems [4]: assuming a simple power system of two voltage buses connected by an inductive power line, the power flow is essentially restricted by a line inductance, even during the transient state. It is therefore useful to examine the stability of VAPAR when it is employed in an RL configuration, estimated so far by a driving point impedance, essentially ignoring the switching process within VAPAR [3].

Two main techniques are available for a more accurate approach: averaging and discrete-time modelling [5]. The drawback of averaging is that as the detailed dynamics within a switching cycle are ignored and only the low-frequency properties retained, the model gives information only about the low-frequency behaviour and its validity will be limited to this range, i.e., it will only be able to predict lowfrequency slow-scale bifurcation behaviour. More complete information can be achieved by sampled-data modelling, using an iterative map that relates the state variables at two consecutive sampling instants [6]. This is the only instrument to detect the bifurcations in the high-frequency range. It is able to predict standard bifurcations and will be used in this study, too, to examine the stability of the RL circuit including a virtual inductance. The stable operation is represented by the periodic regime. All other operations (even though perfectly predictable) are regarded as being undesirable and should be avoided. The results of the analysis demonstrate an interesting practical behaviour of the virtual inductance.

The rest of the paper is organized as follows. In the next section, the configuration and operation of the virtual inductance, employed in an RL circuit, are briefly reviewed. Section 3 develops the Poincaré map and determines the Jacobian matrix belonging to its fixed point. Section 4 presents the calculation results of the characteristic multipliers, used as stability criterion when varying the virtual inductance.

\section{SYSTEM DESCRIPTION}

Figure 1 shows the VAPAR configuration, consisting of an inductor and a voltage source inverter controlled by constantfrequency PWM switching. A feedback loop comprising a PI controller serves to control the virtual flux $\Phi=L_{v} \cdot i_{t}$ of the virtual inductance $L_{v}$, where $i_{t}$ is the terminal current of the VAPAR. On the other hand, the magnetic flux of the inductance follows Faraday's law, therefore the reference flux is obtained as $\Phi_{r e f}=\int v_{t} d t$, where $v_{t}$ is the terminal voltage of the VAPAR. The virtual inductance $L_{v}$ is realized by controlling $\Phi$ to follow $\Phi_{\text {ref. }}$. VAPAR behaves like a twoterminal reactive element and the produced active reactance works as a passive reactive component, including the transient states. It can generate not only positive inductance, but also a negative one, e.g. to be used to cancel an existing undesired inductance [2]. 


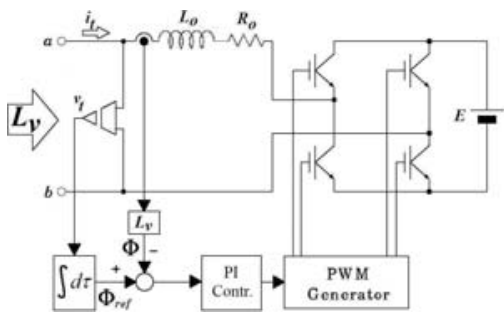

Figure 1: VAPAR configuration

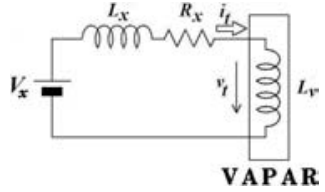

Figure 2: RL circuit including VAPAR
According to the application context mentioned before, the present study is concerned with the stability of this virtual inductance when employed in a series connection with an external inductance $L_{x}$, as shown in Figure 2. For the purpose of simplicity, a DC voltage source $V_{x}$ is considered, but this can also account for an application of VAPAR in a DC link between a rectifier and a current source inverter).

In normal operation the circuit exhibits periodic steadystate behaviour. Periodic time functions (of the PWM switching period) describe the waveforms of the variables, as shown in Figure 3, obtained for $L_{v}=-10 \mathrm{mH}$ and the parameter values listed in Table 1 (the parameters of the PI controller are $K$ and $T_{i}$, the PWM generator employs a ramp waveform of period $T_{s}$ between levels $H$ and $L$ ). The amplitude of the ripple in the voltage $v_{t}$ depends on the ratio of the external inductance $L_{x}$ to the internal inductance $L_{o}$ of the VAPAR. However, it does not affect the operation of VAPAR [2].

\section{THEORETICAL STUDY OF STABILITY}

One of the most useful investigation methods involves a discretization by introducing the Poincaré map [6]. For a non-autonomous periodic system it relates two consecutive points obtained by sampling the state-space trajectory in steady state with the switching period:

$x_{n+1}=P\left(x_{n}\right)$

Since the sampling period $T_{s}$ is the same as the period of the periodic steady-state operation (Figure 3), this regime corresponds to a fixed point $x^{*}$ in the Poincaré map $P$ :

$x^{*}=P\left(x^{*}\right)$

Therefore, the stability of the periodic state is the same as that of the fixed point in the Poincare map, i.e. it is determined by the local behaviour of $P$ near $x^{*}$, governed by its linearization near that fixed point [7]:

$\Delta x_{n+1}=\left.\frac{d P\left(x_{n}\right)}{d x_{n}}\right|_{x^{*}} \Delta x_{n}=J_{P}^{*} \Delta x_{n}=\left[\sum \lambda_{i} u_{i r} u_{i l}^{T}\right] \Delta x_{n}$

The stability of the fixed point is determined by the eigenvalues (characteristic multipliers) $\lambda_{i}$ of the Jacobian matrix $J_{P}^{*}$ of the Poincaré map, evaluated at its fixed point. The fixed point and the periodic operation are asymptotically stable if and only if all characteristic multipliers lie inside the unit circle [8].
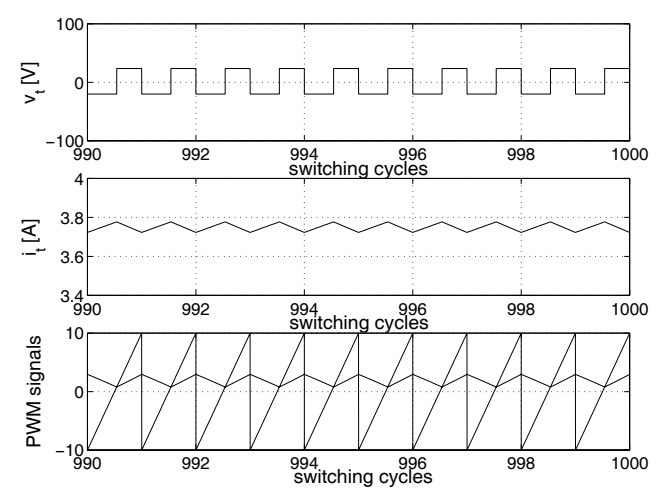

Figure 3: VAPAR terminal voltage (upper), VAPAR terminal current (middle) and PWM comparator signals (lower)

Table1: Parameter and variable values

$V_{x}=30 \mathrm{~V} ; L_{x}=20 \mathrm{mH} ; R_{x}=8 \Omega ; L_{o}=2 \mathrm{mH} ; R_{o}=0.5 \Omega ; E=24 \mathrm{~V}$; $K=4 \times 10^{3} ; T_{i}=1 \mathrm{~ms} ; T_{s}=100 \mu \mathrm{s} ; H=10 ; L=-10$.

The system investigated is variable-structure piecewiselinear. The structure of the active circuit varies during the operation and is depicted in Figure 4a (the state variable $y_{I}$ of the PI controller is also pointed out). Under the constantfrequency PWM switching illustrated in Figure $4 \mathrm{~b}$, the switching function $q(t)$ represents the sign of the difference between the ramp waveform and the signal $y(t)$. The duty ratio $0<d_{n}<1$ of the $n$th cycle is determined by the switching instant $t_{s}$ when they cross within that cycle. Consequently, two periodically toggling linear circuits can be identified during each cycle (Figure 4c). The Poincaré map (1) is determined by solving their state equations: $\dot{x}=A x+B_{1} u$ and $\dot{x}=A x+B_{2} u$, respectively, where

$x=\left[\begin{array}{c}\Phi \\ \Phi_{r e f} \\ y_{I}\end{array}\right] ; u=\left[\begin{array}{c}V_{x} \\ E\end{array}\right] ; A=\left[\begin{array}{ccc}-a & 0 & 0 \\ -b & 0 & 0 \\ -\frac{1}{T_{i}} & \frac{1}{T_{i}} & 0\end{array}\right] ; a=\frac{R_{o}+R_{x}}{L_{o}+L_{x}} ;$

$b=\frac{R_{x} L_{o}-R_{o} L_{x}}{L_{v}\left(L_{o}+L_{x}\right)} ; B_{1}=\left[\begin{array}{cc}l_{v} & l_{v} \\ l_{o} & -l_{x} \\ 0 & 0\end{array}\right] ; B_{2}=\left[\begin{array}{cc}l_{v} & -l_{v} \\ l_{o} & l_{x} \\ 0 & 0\end{array}\right]$;

$l_{v}=\frac{L_{v}}{L_{o}+L_{x}} ; l_{o}=\frac{L_{o}}{L_{o}+L_{x}} ; l_{x}=\frac{L_{x}}{L_{o}+L_{x}}$

The solutions of the two consecutive circuits are:

$x_{s}=W_{1}\left(d_{n}\right) x_{n}+W_{2}\left(d_{n}\right) B_{1} u$

$x_{n+1}=W_{1}\left(1-d_{n}\right) x_{s}+W_{2}\left(1-d_{n}\right) B_{2} u$

where $x_{s}$ is the state vector at the switching instant $t_{s}$, $W_{1}\left(d_{n}\right)=e^{A d_{n} T_{s}}, W_{2}\left(d_{n}\right)=\int_{0}^{d_{n} T_{s}} e^{A \tau} d \tau, W_{1}\left(1-d_{n}\right)=e^{A\left(1-d_{n}\right) T_{s}}$ and $W_{2}\left(1-d_{n}\right)=\int_{0}^{\left(1-d_{n}\right) T_{s}} e^{A \tau} d \tau$ (note that $A$ is singular). 


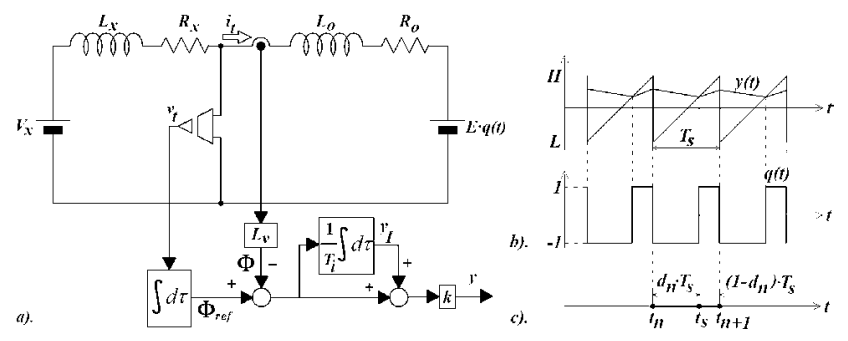

Figure 4: The time-variant system: topology (a), PWM switching (b) and time sequence of structure changes (c)

The Poincaré map (1) is obtained by stacking up the solutions:

$x_{n+1}=P\left(x_{n}, d_{n}\right)=$

$=W_{1}\left(1-d_{n}\right)\left[W_{1}\left(d_{n}\right) x_{n}+W_{2}\left(d_{n}\right) B_{1} u\right]+W_{2}\left(1-d_{n}\right) B_{2} u$

As (7) is a function of $x_{n}$ and $d_{n}$, its Jacobian matrix $J_{P}$ is

$J_{P}\left(x_{n}, d_{n}\right)=\frac{d P\left(x_{n}, d_{n}\right)}{d x_{n}}=\frac{\partial P}{\partial x_{n}}+\frac{\partial P}{\partial d_{n}}\left(\frac{d d_{n}}{d x_{n}}\right)^{T}$

The partial derivatives of $P$ can be calculated from (7) [7]:

$$
\begin{aligned}
& \frac{\partial P}{\partial x_{n}}=W_{1}\left(1-d_{n}\right) W_{1}\left(d_{n}\right) \\
& \frac{\partial P}{\partial d_{n}}=T_{s} W_{1}\left(1-d_{n}\right)\left(\dot{x}_{s 1}-\dot{x}_{s 2}\right)=T_{s} W_{1}\left(1-d_{n}\right)\left(B_{1}-B_{2}\right) u
\end{aligned}
$$

where $\dot{x}_{s 1}$ and $\dot{x}_{s 2}$ are the velocities of the state vector at the end of first structure and at the beginning of the second one, respectively.

The relation between the duty ratio $d_{n}$ of the cycle and the state vector $x_{n}$ at the beginning of that cycle is needed in order to determine the derivative of $d_{n}$ in (8). The PWM control ends the first structure at the switching instant $t_{s}$, when the difference between the signal $y$ and the ramp waveform becomes zero (Figure 4b,c):

$$
\begin{aligned}
& e\left(x_{n}, d_{n}\right)=y\left(t_{s}\right)-L-(H-L) d_{n}= \\
& \quad=c^{T}\left[W_{1}\left(d_{n}\right) x_{n}+W_{2}\left(d_{n}\right) B_{1} u\right]-L-(H-L) d_{n}=0
\end{aligned}
$$

where $c^{T}=K \cdot\left[\begin{array}{lll}-1 & 1 & 1\end{array}\right]$.

For small changes $\left(\partial e / \partial x_{n}\right)^{T} d x_{n}+\left(\partial e / \partial d_{n}\right) d d_{n}=0$ and

$\left(\frac{d d_{n}}{d x_{n}}\right)^{T}=-\left(\frac{\partial e}{\partial d_{n}}\right)^{-1}\left(\frac{\partial e}{\partial x_{n}}\right)^{T}$

The two partial derivatives are obtained from (11):

$$
\begin{aligned}
& \frac{\partial e}{\partial d_{n}}=c^{T} \dot{x}_{s 1} T_{s}-(H-L)=c^{T}\left(A x_{s}+B_{1} u\right) T_{s}-(H-L) \\
& \left(\frac{\partial e}{\partial x_{n}}\right)^{T}=c^{T} W_{1}\left(d_{n}\right)
\end{aligned}
$$

Substituting (13) into (8), the Jacobian matrix is:

$J_{P}\left(x_{n}, d_{n}\right)=\frac{\partial P}{\partial x_{n}}-\frac{\partial P}{\partial d_{n}}\left(\frac{\partial e}{\partial d_{n}}\right)^{-1}\left(\frac{\partial e}{\partial x_{n}}\right)^{T}$

For the fixed point $x^{*}$ and the corresponding duty ratio $d^{*}$, by substituting all the partial derivatives (9), (10), (14), (15):

$$
J_{P}^{*}=W_{1}\left(1-d^{*}\right)\left[I-\frac{\left(B_{1}-B_{2}\right) u c^{T}}{c^{T}\left(A x_{s}^{*}+B_{1} u\right)-\frac{H-L}{T_{s}}}\right] W_{1}\left(d^{*}\right)
$$

where $I$ denotes the identity matrix.

The calculation of the Jacobian matrix in (17) requires the duty ratio $d^{*}$ in periodic steady state and the corresponding state vector $x_{s}^{*}$ at the switching instant within a cycle. They can be related from (5) and (6) after substituting the fixed point $x^{*}=x_{n}=x_{n+1}$ :

$\left[I-W_{1}(1)\right] x_{s}^{*}=\left[W_{1}\left(d^{*}\right) W_{2}\left(1-d^{*}\right) B_{2}+W_{2}\left(d^{*}\right) B_{1}\right] u$

(18) does not provide $x_{s}^{*}$ as a function of $d^{*}$ since

$$
I-W_{1}(1)=\left[\begin{array}{ccc}
1-e^{-a T_{s}} & 0 & 0 \\
\frac{b}{a}\left(1-e^{-a T_{s}}\right) & 0 & 0 \\
{\left[\left(1-\frac{b}{a}\right) \frac{1-e^{-a T_{s}}}{a T_{s}}+\frac{b}{a}\right] \frac{T_{s}}{T_{i}}} & -\frac{T_{s}}{T_{i}} & 0
\end{array}\right]
$$

is singular. However, its first two rows are proportionally related, therefore multiplying (18) from left with $v_{1}^{T}=\left[\begin{array}{lll}-b & a & 0\end{array}\right]$ yields:

$0=v_{1}^{T}\left[W_{1}\left(d^{*}\right) W_{2}\left(1-d^{*}\right) B_{2}+W_{2}\left(d^{*}\right) B_{1}\right] u$

which can be numerically solved for $0 \leq d^{*} \leq 1$.

(12) relates $d^{*}$ and $x_{s}^{*}$ as well:

$c^{T} x_{s}^{*}=L+(H-L) d^{*}$

The matrix equation built from the last two rows of (18) and (21) as third row can be solved explicitly for $x_{s}^{*}$ since its coefficient is no longer singular:

$$
\begin{aligned}
x_{s}^{*}= & \left\{M\left[I-W_{1}(1)\right]+v_{2} c^{T}\right\}^{-1}\left\{M \left[W_{1}\left(d^{*}\right) W_{2}\left(1-d^{*}\right) B_{2}\right.\right. \\
& \left.\left.+W_{2}\left(d^{*}\right) B_{1}\right] u+v\left[L+(H-L) d^{*}\right]\right\}
\end{aligned}
$$

where $M=\left[\begin{array}{lll}0 & 1 & 0 \\ 0 & 0 & 1 \\ 0 & 0 & 0\end{array}\right]$ and $v_{2}=\left[\begin{array}{l}0 \\ 0 \\ 1\end{array}\right]$.

Having computed $d^{*}$ from (20) and $x_{s}^{*}$ from (22), the Jacobian (17) and its eigenvalues can be calculated numerically.

\section{RESULTS OF STABILITY ANALYSIS}

In order to find out the bifurcation scenarios, loci of the eigenvalues are presented in Figure 5 as the virtual inductance $L_{v}$ is varied (the other parameter values are listed in Table 1). According to the stability condition, as long as the characteristic multipliers lie inside the unit circle, the periodic steady-state solution is asymptotically stable. As the positive virtual inductance is increased, a real characteristic multiplier moves out of the unit circle through -1 , indicating a period-doubling bifurcation: the periodic solution with period $T_{s}$ changes into one with period $2 T_{s}$ (Figure 6). As the 
negative virtual inductance is decreased, a complex-conjugate pair of characteristic multipliers moves out of the unit circle denoting a Neimark-Sacker bifurcation: the periodic solution loses stability and a quasi-periodic one is generated (Figure 7). Previous studies have already conditioned the stability of the negative virtual inductance by $L_{v}+L_{x}>0$ [2]. The results of this study are more restrictive and they depend on other parameters, too, e.g., for $V_{x}=10 \mathrm{~V}$ the stability of the negative virtual inductance is lost through the same NeimarkSacker bifurcation at a lower value, $L_{v}=-19 \mathrm{mH}$. As presented, simulations confirmed the results of this analytical stability study.
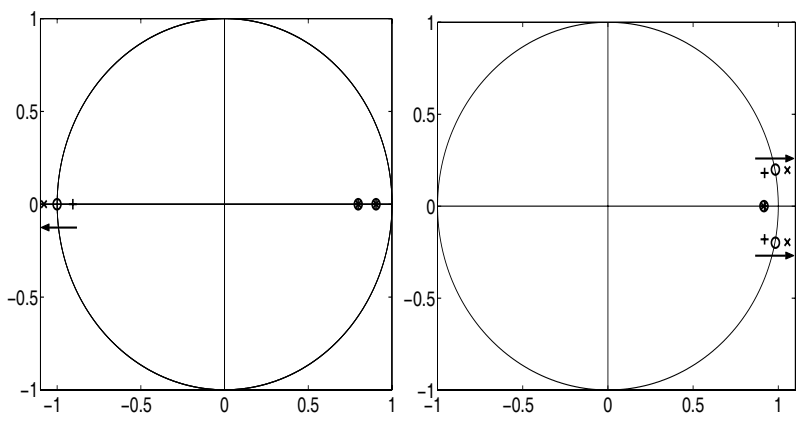

Figure 5: Loci of characteristic multipliers for increasing positive (left): $L_{v}=300 \mathrm{mH}$ ('+'), $L_{v}=470 \mathrm{mH}$ ('o'), $L_{v}=800 \mathrm{mH}$

(' $\mathrm{x}$ ') and decreasing negative (right): $L_{v}=-13 \mathrm{mH}$ ('+'), $L_{v}=-16 \mathrm{mH}$ ('o'), $L_{v}=-19 \mathrm{mH}$ ('x') virtual inductance

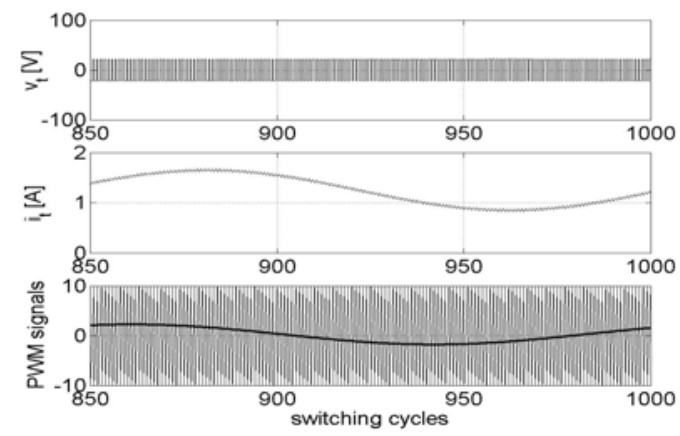

Figure 6: Quasi-periodic VAPAR terminal voltage (upper) and current (middle), PWM comparator signals (lower)

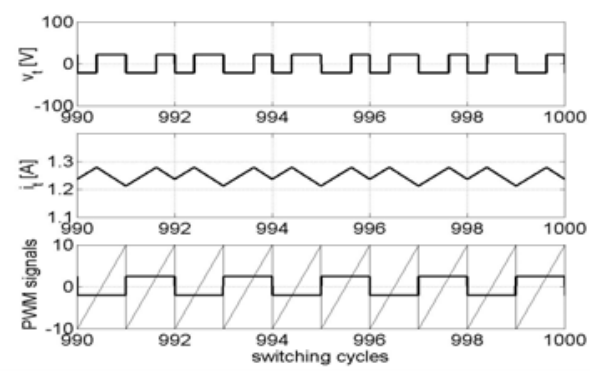

Figure 7: Period-doubled VAPAR terminal voltage (upper) and current (middle), PWM comparator signals (lower)

\section{CONCLUSION}

This paper has reported the stability investigation of an RL circuit including a variable active-passive reactance that can generate a virtual inductance, including a negative one. The periodic steady-state operation of this variable-structure piecewise-linear nonlinear system is modeled by its Poincaré map. The stability analysis determines the characteristic multipliers of the Jacobian matrix of the Poincaré map, evaluated at its fixed point. It demonstrates a bifurcation behaviour which has not been previously detected. By increasing the positive virtual inductance, the stability is lost through a period-doubling bifurcation, when a real characteristic multiplier moves out of the unit circle through -1 . By decreasing the negative virtual inductance, the stability is lost when a complex-conjugate pair of characteristic multipliers moves out of the unit circle and a quasi-periodic state develops through a Neimark-Sacker bifurcation. The analysis facilitates a more accurate selection of parameter values to guarantee stable operation.

\section{REFERENCES}

[1] H. Funato and A. Kawamura, "Proposal of Variable ActivePassive Reactance," International Conference on Industrial Electronics, Control, Instrumentation and Automation (IECON'92), San Diego, USA, pp. 381-388, Nov. 1992.

[2] H. Funato, A. Kawamura and K. Kamiyama, "Realization of Negative Inductance Using Variable Active-Passive Reactance (VAPAR)," IEEE Transactions on Power Electronics, Vol. 12, No. 4, pp. 589-596, July 1997.

[3] H. Funato, K. Kamiyama and A. Kawamura, "Transient Performance of Power Circuit Including Virtual Inductance Realized by Fully Digital Controlled Variable Active-Passive Reactance (VAPAR)," Power Electronics Specialists Conference, Galway, Ireland, pp. 1195-1200, June 2000.

[4] H. Funato, K. Kamiyama and A. Kawamura, "A New Instantaneous Power Flow Control Method Using Variable Inductance Realized by Variable Active-Passive Reactance (VAPAR)," Trans. IEE of Japan, Vol. 120-D, No. 10, pp. 12051211, Oct. 2000.

[5] S. Banerjee and G. Verghese (eds.), Nonlinear Phenomena in Power Electronics, IEEE Press, New York, April 2001.

[6] I. Nagy, "Nonlinear Phenomena in Power Electronics," Automatika, vol. 42, no. 3-4, pp. 117-132, July-Dec. 2001.

[7] O. Dranga, B. Buti and I. Nagy, "Stability Analysis of a Feedback-Controlled Resonant DC-DC Converter," IEEE Trans on Ind. Electronics, Vol.50, No.1, pp. 141-152, February 2003.

[8] H.H.C. Iu, C.K. Tse, V. Pjevalica and Y.M. Lai, "Bifurcation Behaviour in Parallel-Connected Boost Converters," International Journal of Circuit Theory and Applications, Vol. 29, pp. 281-298, May 2001. 